\title{
Detection of expiratory flow limitation in COPD using the forced oscillation technique
}

\author{
R.L. Dellacà*, P. Santus" ${ }^{\#}$ A. Aliverti*, N. Stevenson , S. Centanni” , P.T. Macklem ${ }^{+}$, A. Pedotti*,
P.M.A. Calverley
}

Detection of expiratory flow limitation in COPD using the forced oscillation technique. R.L. Dellacà, P. Santus, A. Aliverti, N. Stevenson, S. Centanni, P.T. Macklem, A. Pedotti, P.M.A. Calverley. (C) ERS Journals Ltd 2004.

ABSTRACT: Expiratory flow limitation (EFL) during tidal breathing is a major determinant of dynamic hyperinflation and exercise limitation in chronic obstructive pulmonary disease (COPD). Current methods of detecting this are either invasive or unsuited to following changes breath-by-breath. It was hypothesised that tidal flow limitation would substantially reduce the total respiratory system reactance $\left(\mathrm{Xrs}_{\mathrm{rs}}\right)$ during expiration, and that this reduction could be used to reliably detect if EFL was present.

To test this, $5-\mathrm{Hz}$ forced oscillations were applied at the mouth in seven healthy subjects and 15 COPD patients (mean \pm SD forced expiratory volume in one second was $36.8 \pm 11.5 \%$ predicted) during quiet breathing. COPD breaths were analysed $(n=206)$ and classified as flow-limited if flow decreased as alveolar pressure increased, indeterminate if flow decreased at constant alveolar pressure, or nonflow-limited.

Of these, 85 breaths were flow-limited, 80 were not and 41 were indeterminate. Among other indices, mean inspiratory minus mean expiratory $X_{\mathrm{rss}}\left(\Delta \overline{X_{\mathrm{rs}}}\right)$ and minimum expiratory $X_{r s}(X e x p, m i n)$ identified flow-limited breaths with $100 \%$ specificity and sensitivity using a threshold between $2.53-3.12 \mathrm{cmH}_{2} \mathrm{O} \cdot \mathrm{s} \cdot \mathrm{L}^{-1}\left(\Delta \overline{X_{\mathrm{rs}}}\right)$ and $-7.38--6.76 \mathrm{cmH}_{2} \mathrm{O} \cdot \mathrm{s} \cdot \mathrm{L}^{-1}$ (Xexp,min) representing 6.0\% and 3.9\% of the total range of values respectively. No flow-limited breaths were seen in the normal subjects by either method.

Within-breath respiratory system reactance provides an accurate, reliable and noninvasive technique to detect expiratory flow limitation in patients with chronic obstructive pulmonary disease.

Eur Respir J 2004; 23: 232-240.
*TBMLab, Dipartimento di Bioingegneria, Politecnico di Milano University and ${ }^{\#}$ Respiratory Unit, Institute of Lung Disease, San Paolo Hospital, University of Milan, Milan, Italy. "University Hospital Aintree, University Dept of Medicine, Liverpool, UK. ${ }^{+}$Respiratory Division, Meakins-Christie Laboratories, MUHC Research Institute, McGill University, Montreal, Canada.

Correspondence: R.L. Dellacà, Dipartimento di Bioingegneria, Politecnico di Milano, Piazza Leonardo da Vinci 32, I-20133 Milan, Italy. Fax: 390223999000

E-mail: dellaca@biomed.polimi.it

Keywords: Chronic obstructive pulmonary disease, forced oscillation technique, impedance, respiratory reactance, within-breath reactance

Received: April 282003

Accepted after revision: October 72003

This work was supported by the European Community CARED FP5 Project (Contract number QLRT-2001-0893).
Unlike healthy subjects who do not develop expiratory flow limitation (EFL) even during exhaustive exercise [1], many chronic obstructive pulmonary disease (COPD) patients are flow-limited (FL) at rest [2]. These patients can only increase their expiratory flow rate during exercise by allowing their end-expiratory lung volume $(V \mathrm{~L})$ to rise, an energetically inefficient strategy that is accompanied by severe dyspnoea that reduces exercise duration [3, 4]. The severity of dyspnoea in COPD is better predicted by the presence of EFL during tidal breathing than by the forced expiratory volume in one second (FEV1) [5, 6]. Thus, a simple method of detecting EFL during tidal breathing would be a potentially useful clinical tool. Several noninvasive methods have been proposed to detect tidal EFL in COPD patients, but each has its limitations and, to the best of the authors' knowledge, to date none has been tested against any form of "gold standard" in spontaneously breathing patients.

In 1993, PESLIN et al. [7] reported that some COPD patients during mechanical ventilation developed large negative swings in the respiratory system input reactance (Xrs, i.e. the imaginary part of total input impedance) measured by a forced oscillation technique (FOT). Similar behaviour was observed in a simplified mechanical model of the respiratory system when a flow-limiting segment was included [8] and in mechanically

For editorial comments see page 187. ventilated rabbits [9] after intravenous methacholine infusion. This phenomenon occurs because the linear velocity of gas passing through flow-limiting segments (choke points) equals the local speed of wave propagation [10]. Normally the reactance reflects the elastic and inertial properties of the respiratory system but when flow limitation is present, the oscillatory signal cannot pass through the choke points and reach the alveoli, producing a marked reduction in the apparent compliance (and, consequently, a fall in Xrs). These theoretical and experimental considerations make within-breath reactance measurement a potentially useful indicator of the occurrence of tidal EFL in COPD.

The authors hypothesised that the decrease of withinbreath Xrs during expiration would allow the definition of a sensitive and specific method of determining the presence of EFL. To confirm this, breaths with and without a decrease in expiratory flow were studied while alveolar pressure $(P \mathrm{~A})$ increased, an independent way of identifying the presence of EFL in spontaneously breathing subjects.

\section{Methods}

\section{Subjects}

Fifteen stable COPD patients and seven age-matched healthy subjects were studied whose characteristics and lung 


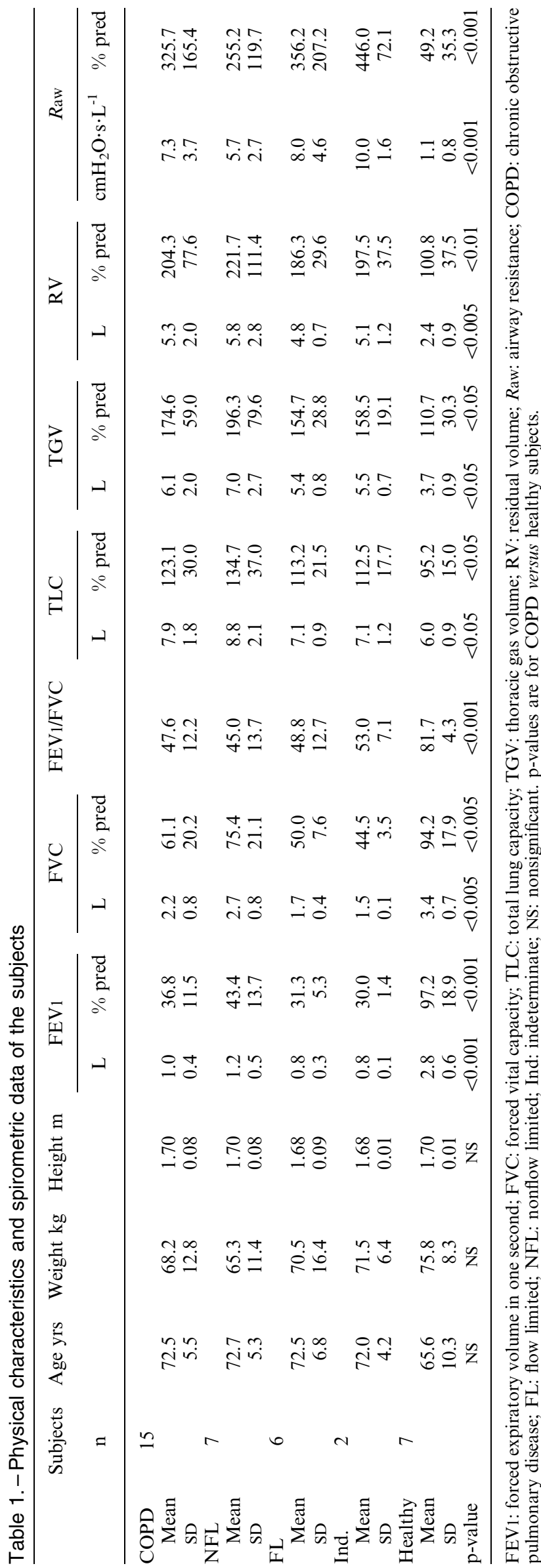

function are shown in table 1 . The patients met the standard diagnostic criteria for COPD [11] and were current or exsmokers. They omitted their short- or long-acting bronchodilators for $\geqslant 3$ and $\geqslant 12 \mathrm{~h}$, respectively, before the study. Spirometry and subdivisions of $V \mathrm{~L}$ were measured in a constant-volume body plethysmograph (Medgraphic Autolink 1085D, Medical Graphics, St Paul, MN, USA). Predicted values for flows and volumes were those recomended by the European Respiratory Society [12]. FOT was applied to the mouth of each subject while seated, wearing a noseclip and mouthpiece, during 2-3 min of spontaneous breathing. An operator firmly supported the cheeks to reduce upper airways shunt. The study was approved by the institutional research ethics committee, and written informed consent was given by each subject.

\section{Measurements}

Pressure $(P$ ao $)$ and flow $\left(V^{\prime}\right.$ ao $)$ at the airway opening were measured by a transducer (SCX01, SenSym, Milpitas, CA, USA) connected to the mouthpiece and by a screen-type pneumotachograph (4700A; Hans Rudolph, Kansas City, MO connected to a transducer LCVR, 0-2 $\mathrm{cmH}_{2} \mathrm{O}$; Celesco Instruments, Canoga Park, CA). Oesophageal pressure ( $P$ oes) was measured by a pressure transducer (SCX05, Sensym) connected to a standard balloon-catheter system placed in the lower oesophagus and filled with $0.4 \mathrm{~mL}$ of air. The position of the balloon was confirmed using the occlusion method [13]. All the signals were sampled at $200 \mathrm{~Hz}$ by an analogue-todigital and digital-to-analogue board (DAQ-CARD 1200; National Instruments, Austin, TX) and recorded by a personal computer. The flow signal was integrated to give $V \mathrm{~L}$. The frequency response of the measuring systems [14] was flat up to $30 \mathrm{~Hz}$.

\section{Forced oscillations}

The experimental set-up for FOT measurement is shown in figure 1. Healthy subjects and patients were studied while being oscillated by $5 \mathrm{~Hz}$ sinusoidal forcing with a pressure amplitude at the mouth of $\sim 2 \mathrm{cmH}_{2} \mathrm{O}$. The forcing frequency was chosen based on the preliminary model simulations presented in the Appendix. The same computer and board used to sample flow and pressure signals generated the forcing signal, which was amplified by a power amplifier (Proline EQ552; Eurosound, Milan, Italy) connected to a $25-\mathrm{cm}$ diameter loudspeaker (HS250; Ciare, Ancona, Italy) mounted on a rigid box of $\sim 2 \mathrm{~L}$ of internal volume. The pressure generated by the loudspeaker was transferred from the box through a connecting tube $(22 \mathrm{~cm}$ in length, $19 \mathrm{~mm}$ in internal diameter) to the subject's mouthpiece. A low-resistance, high-inertance tube $(35 \mathrm{~mm}$ in internal diameter and $1.5 \mathrm{~m}$ in length) in parallel with the loudspeaker allowed the subjects to breathe room air without significant loss of forcing pressure. A bias flow of $\sim 15 \mathrm{~L} \cdot \mathrm{min}^{-1}$ reduced the equipment deadspace to the volume of the pneumotachograph and the mouthpiece [15].

\section{Detection of expiratory flow limitation by the Mead and Whittenberger method}

The method of MEAD and WhitTENBerger [16] (M-W) of measuring pulmonary resistance was used to detect EFL during tidal breathing simultaneously with the application of forced oscillation. Briefly, the flow-resistive pressure (Pfr; 
equal to $P$ ao $-P$ A) along the tracheobronchial tree was estimated by subtracting the elastic recoil pressure of the lung from transpulmonary pressure. During quiet breathing, elastic recoil pressure is directly proportional to volume. Thus a signal proportional to volume was subtracted from transpulmonary pressure. The constant of proportionality was adjusted so that the pressure at zero flow points at the beginning and end of inspiration were identical. Using zeroflow points to estimate $P$ fr the inertial pressure, even if very small during normal breathing, is neglected. Also lung tissue resistance, which may introduce a pressure drop between the pleura and the alveoli, and possible within-breath changes in upper airway resistance are neglected by the $\mathrm{M}-\mathrm{W}$ method.

When the Lissajous figure in the Pfr versus flow graphs (fig. 2a-c) showed a loop where flow decreased during expiration while $P$ fr increased the breath was classified as "flow-limited" (FL; fig. 2c). Conversely, if the expiratory phase was characterised by a quasi-linear dependence between $P$ fr and flow with little or no loop the breath was classified as "nonflow-limited" (NFL; fig. 2b). In cases where it was not possible to be certain if flow limitation was present the breaths were classified as "indeterminate". This occurred in two different circumstances: when the inspiratory pressure/ flow curve was looped instead of closed (possible errors in elastic recoil pressure estimation or an expiratory loop produced by the changes of $V \mathrm{~L}$ and not by EFL) or when the expiratory pressure flow curve was characterised by a clockwise loop in which flow decreased but $P$ fr did not simultaneously increase significantly.

\section{Data analysis}

Within-breath input impedance (Zin; and thus Xrs) was determined by using a least squares algorithm $[17,18]$ taking

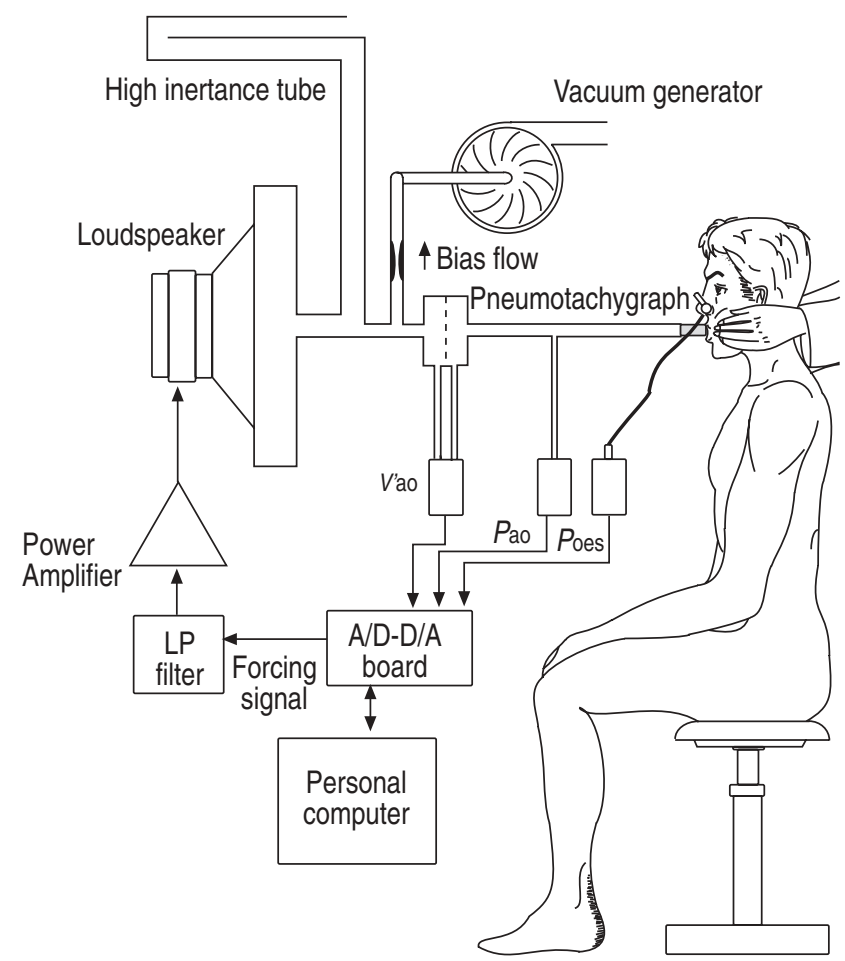

Fig. 1.-Experimental set-up for within-breath impedance measurement. $V^{\prime}$ ao: flow at airway opening; $P$ ao: pressure at airway opening; Poes: oesophageal pressure; LP: low pass; A/D-D/A: analogue-todigital and digital-to-analogue. advantage of the a priori knowledge of the frequency spectrum components of the forcing signals. This method measures the input impedance for every acquired sample using a moving time window of pressure and flow signals of $0.2 \mathrm{~s}$.

From the quiet breathing tracings, the longest period in which the breathing pattern was stable and without oesophageal spasms was selected. Four different indices based on the anticipated reactance change were used to detect EFL: 1) the mean value of Xrs during expiration $(\bar{X} \exp ) ; 2)$ the minimal value of Xrs during expiration (Xexp,min); 3) the difference between the mean value of $\mathrm{Xrs}$ during inspiration ( $\left.\bar{X}_{\text {insp }}\right)$ and $\bar{X} \exp (\Delta \overline{X r s})$; and 4$)$ the difference between the maximal value of $\mathrm{Xrs}$ during inspiration (Xinsp,max) and Xexp,min (Xpeak-to-peak; fig. 3).

Different thresholds were applied to the values of each index ( $\bar{X} \exp , \mathrm{X}$ exp,min, $\Delta \bar{X}_{\mathrm{rs}}$ and Xpeak-to-peak) computed breath-by-breath. All breaths classified unequivocally by the $\mathrm{M}-\mathrm{W}$ analysis as either FL or NFL were used to determine the sensitivity (the number of detected FL breaths divided by the total number of FL breaths) and specificity (the number of detected NFL breaths divided by the total number of NFL breaths) of each index. Sensitivity and specificity were calculated for the range of possible threshold values for each index in the following way: the total range of values assumed by an index was subdivided into 100 equally spaced points to provide a set of possible threshold values with good resolution. Then sensitivity and specificity were computed for each of the 100 values. These data were plotted as a function of the threshold value on the same graph. Areas where sensitivity and specificity curves were both $100 \%$ defined the optimal range of threshold values. Optimal threshold was chosen as the midpoint of this range.

Significance of differences of physical characteristics, spirometric data and Xrs indices' values between different groups was performed by a nonparametric (Mann-Whitney) test. Data are expressed as mean \pm SD unless otherwise stated.

\section{Results}

Representative Xrs data are presented in the lower panels of figure 2 where three experimental tracings of $P \mathrm{fr}$ and $\mathrm{Xrs}$ obtained during a quiet breath are shown for a control, an NFL COPD patient and an FL COPD patient. The Pfr versus flow curve for the same breath is shown in the upper panel. Clear differences can be observed between the inspiratory and expiratory reactance in the patient where flow limitation was present ( $\mathrm{c}$ and $\mathrm{f}$ ) but not in the other examples.

In figure 3 the experimental tracings of volume, flow, pressure, total respiratory input resistance (Rrs) and Xrs are shown for a representative FL COPD patient. Note that the Rrs time course, unlike that for Xrs, did not present clear differences between inspiration and expiration. The withinbreath fluctuations of Rrs were usually wider if EFL was present than in the absence of flow limitation, a finding common to most of the breaths studied. These results are in agreement with the model data presented in the Appendix. Of the 284 breaths (206 from patients and 78 from controls) selected, 12 breaths (4\%) were discarded because of oesophageal spasms, spikes in the impedance due to glottis closure or swallowing, or because they showed an abnormal looping of the pressure/flow curve. The authors used 85 breaths classified unequivocally by $\mathrm{M}-\mathrm{W}$ analysis as FL and 80 as NFL to determine sensitivity and specificity of the indices. Sensitivity and specificity plots are presented as function of the threshold value for each index (fig. 4). All indices had a region where both specificity and sensitivity were $100 \%$ but these regions 

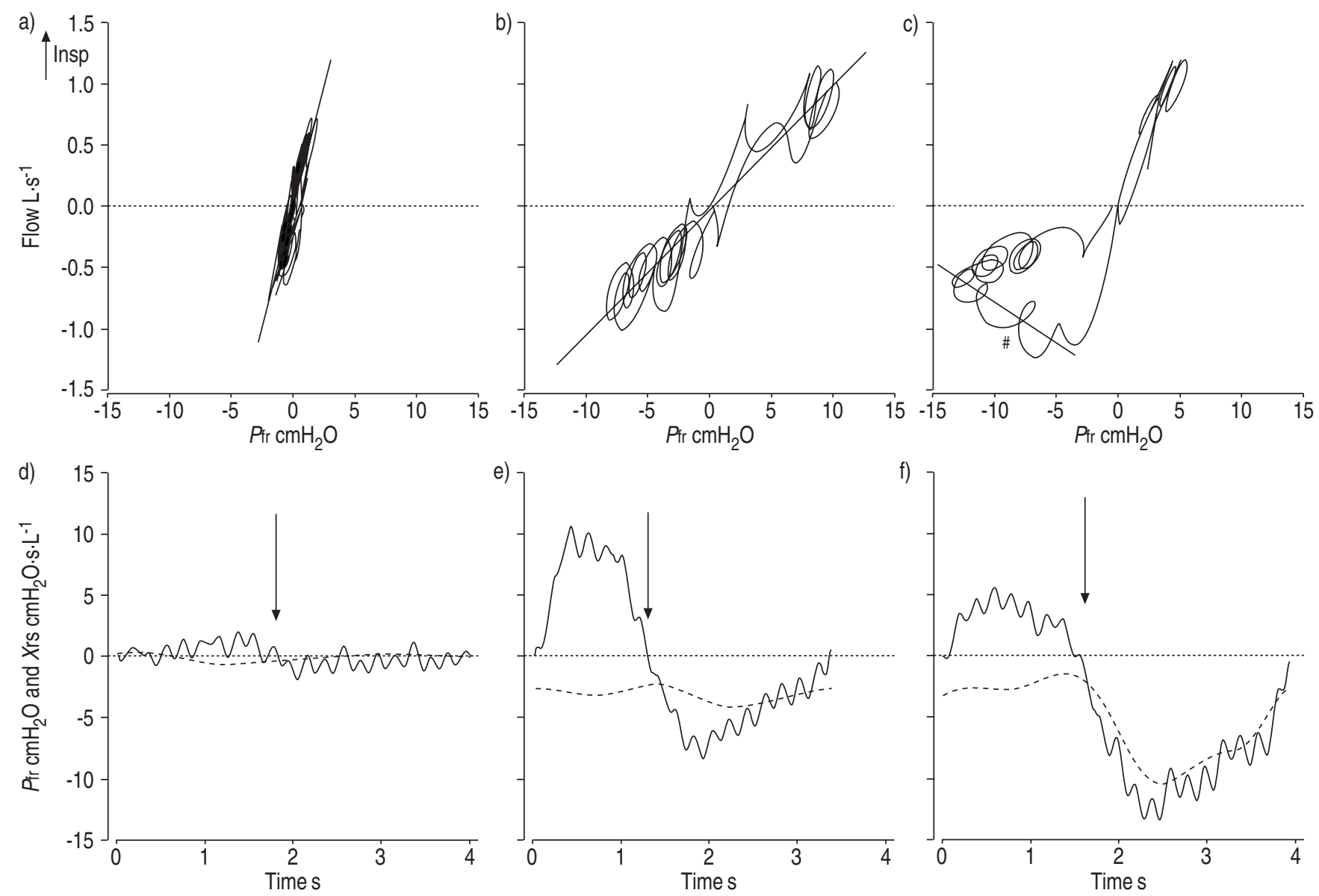

Fig. 2. - The Mead and Whittenberger graphs $(\mathrm{a}-\mathrm{c})$ obtained by plotting the airway opening flow versus the resistive pressure drop $(P$ fr $)$ during a single breath. Data from a healthy subject (a and d), a nonflow-limited patient (b and e) and a flow-limited chronic obstructive pulmonary disease patient ( $\mathrm{c}$ and $\mathrm{f}$ ) are shown. The regression lines in a) $\left(\mathrm{m}=0.398, \mathrm{R}=1 / \mathrm{m}=2.51 \mathrm{cmH}_{2} \mathrm{O} \cdot \mathrm{s} \cdot \mathrm{L}^{-1}\right)$ and $\left.\mathrm{b}\right)\left(\mathrm{m}=0.102, \mathrm{R}=1 / \mathrm{m}=9.8 \mathrm{cmH} \mathrm{H}_{2} \mathrm{O} \cdot \mathrm{s} \cdot \mathrm{L}^{-1}\right)$ represent airway resistance at breathing frequency, $\mathrm{m}$ being the slope of the regression (airway conductance). In c), expiratory flow limitation (\#) is demonstrated by the presence of a region in which airway opening flow is decreasing while $P$ fr is increasing. The lower graphs (d-f) show the corresponding time courses of $\operatorname{Pfr}(-)$ and respiratory system reactance (- - ). The arrows indicate the end of inspiration i.e. time before this point is inspiration and after is expiration.

had different widths. Total range, optimal range (range of threshold values in which both specificity and sensitivity were $100 \%$ ), its percentage of the total range (optimal region) and the midpoint of the optimal range (optimal threshold) are shown in table 2 for each index.

The patients were then divided into three groups depending on the classification of their breaths analysed by the M-W. In 11 patients, all of the breaths were within the same classification, but in four patients different breaths were classified in different categories. However, in these patients there was always a clear majority of breaths (75\% minimum) in the same category. Thus, six patients were classified as FL during tidal ventilation, seven patients as NFL and two patients as indeterminate.

In general, patients with more severe COPD were more likely to be FL, as would be expected. However, even if the FL patients presented in average a lower value of FEV1 than NFL (see table 1), the lowest FEV1 showed by NFL patients ( $25 \%$ predicted) was much smaller than the highest presented by FL patients $(41 \%$ pred).

Mean values of all the indices for each patient and control subject are presented in figure 5 as well as the average values for each group. As expected there was a clear distinction between the FL and NFL groups with the two indeterminate patients presenting values similar to the FL patients. Healthy subjects presented values closer (even if statistically different) to NFL COPD patients.

The application of the threshold values determined in COPD patients to the indices computed for the healthy subjects indicates that in healthy subjects EFL was never present during quiet breathing, with values for all the indices clearly separated from the optimal threshold (horizontal dashed line in figure 5) selected from table 2.

\section{Discussion}

The ability to detect EFL reliably and noninvasively during tidal breathing is of both theoretical and practical value in patients with COPD. Current techniques all have significant disadvantages, although more recently developed methods based on detecting changes in expiratory flow when the driving pressure is increased are more convenient and simpler to apply than previous approaches $[19,20]$. However, even these methods are limited in the number of breaths that can be tested and/or by the need to perform a specific respiratory manoeuvre. Moreover, none has been compared with an independent method to detect EFL during awake spontaneous breathing subjects

By definition, flow limitation occurs when maximum 


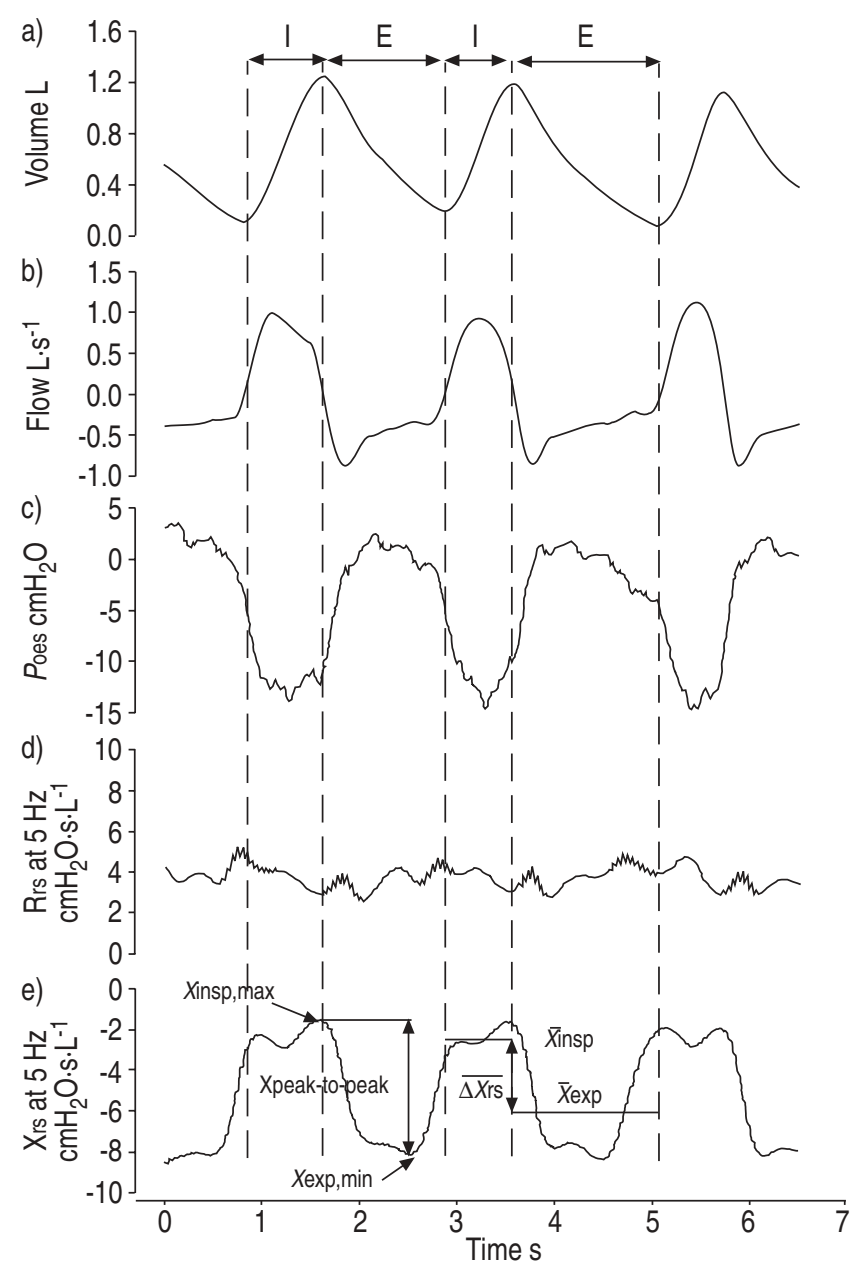

Fig. 3.-Experimental tracing from a representative flow-limited patient and definition of the indices used to characterise the respiratory system reactance $\left(\mathrm{X}_{\mathrm{rs}}\right)$ time course during a single breath. a) Respiratory volume, b) flow at the airway opening, c) oesophageal pressure, d) total respiratory input resistance (Rrs) and d) $\mathrm{Xrs}_{\mathrm{rs}}$ at $5 \mathrm{~Hz}$. $X$ insp: mean value of $X_{\text {rs }}$ during inspiration; $X_{\text {exp: mean }}$

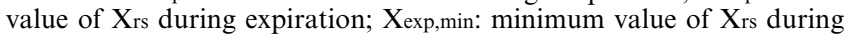
expiration; $X$ insp,max: maximum value of $X_{r s}$ during inspiration. Since reactance was expected to decrease during expiratory flow limitation, the difference between $\bar{X}_{\text {insp }}$ and $\bar{X}_{\exp }\left(\Delta \overline{X_{\mathrm{rs}}}\right)$ and the difference between Xinsp,max and Xexp,min (Xpeak-to-peak) was considered. Indices were defined in two different breaths for clarity. I: inspiration; E: expiration; Poes: oesophageal pressure.

expiratory flow is reached on the plateau of the isovolume pressure/flow curves [21]. Unfortunately this approach is not suitable for a simultaneous comparison with the measurement of Xrs. Therefore, flow limitation was defined as a decrease in
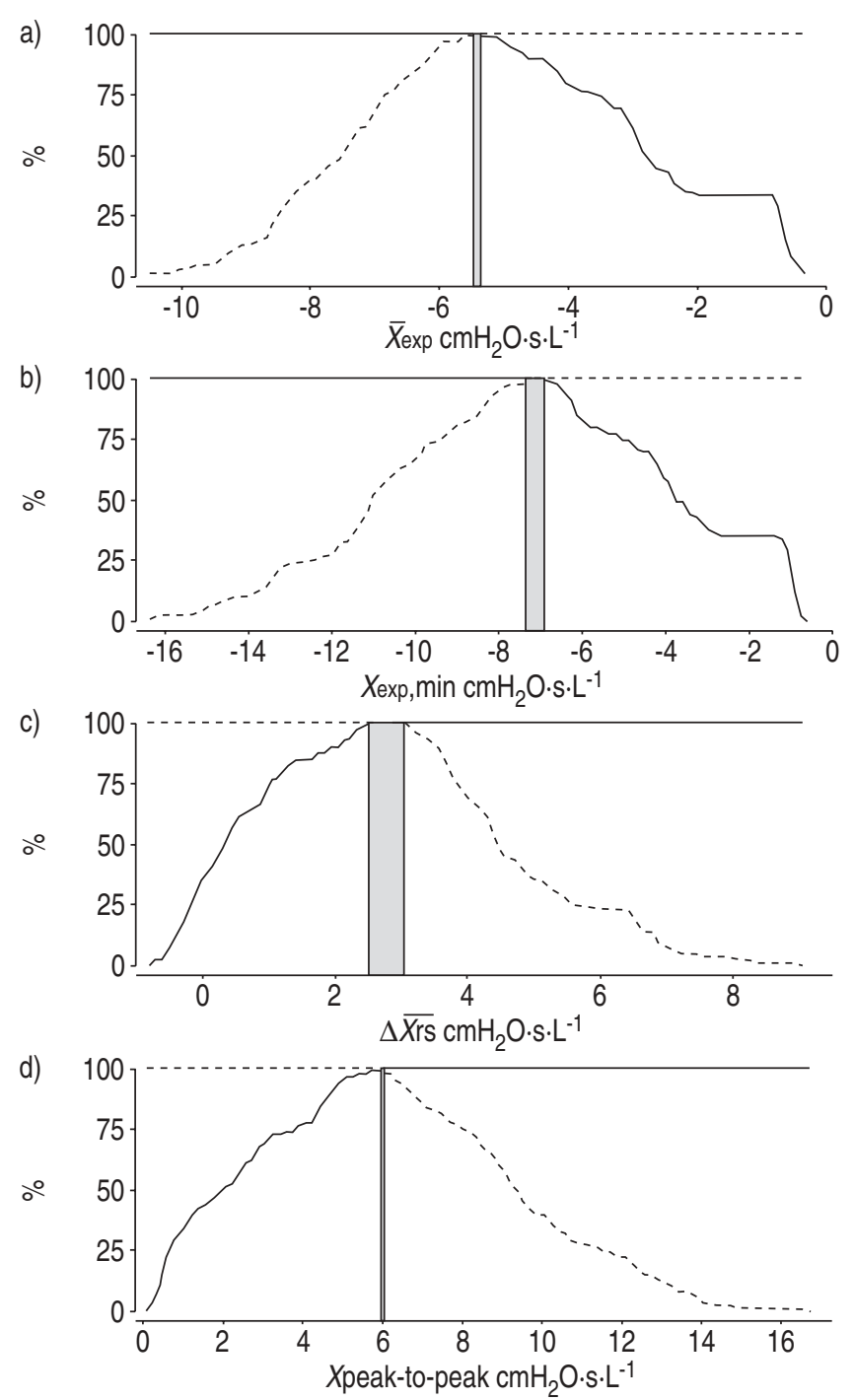

Fig. 4. - Sensitivity (- - -) and specificity (-) expressed as percentage of all the classifiable breaths ( 80 nonflow-limited and 85 flow-limited) are plotted versus the threshold values for the four considered indices. a) Mean expiratory $\mathrm{X}_{\mathrm{rs}}$ value $\left(\bar{X}_{\mathrm{exp}}\right)$, b) minimum expiratory $\mathrm{X}_{\mathrm{rs}}$ value $(X$ exp, min), c) the difference between mean inspiratory and mean expiratory $\mathrm{X}_{\mathrm{rs}}\left(\Delta \overline{X_{\mathrm{rs}}}\right)$ and $\left.\mathrm{d}\right)$ the difference between maximum inspiratory and minimum expiratory $\mathrm{X}_{\mathrm{rs}}$ (X $\mathrm{X}_{\text {peak-to-peak }}$ ) were considered. The shaded bars represent the optimal region in which sensitivity and specificity are both $100 \%$.

$V^{\prime}$ ao with an increase in $P$ fr. This is essentially the same definition used in the negative expiratory pressure (NEP) technique; when a negative pressure is applied to the airway

Table 2. - Total range, optimal range, region width and threshold for respiratory system reactance (Xrs) indices in patients

\begin{tabular}{lcccc}
\hline Index & $\begin{array}{c}\text { Total range } \\
\mathrm{cmH} \mathrm{H}_{2} \mathrm{O} \cdot \mathrm{s} \cdot \mathrm{L}^{-1}\end{array}$ & $\begin{array}{c}\text { Optimal range } \\
\mathrm{cmH} \mathrm{H}_{2} \mathrm{O} \cdot \mathrm{s} \cdot \mathrm{L}^{-1}\end{array}$ & $\begin{array}{c}\text { Optimal } \\
\text { region \% }\end{array}$ & $\begin{array}{c}\text { Optimal threshold } \\
\mathrm{cmH} \mathrm{H}_{2} \mathrm{O} \cdot \mathrm{s} \cdot \mathrm{L}^{-1}\end{array}$ \\
\hline $\bar{X}_{\text {exp }}$ & $-10.5--0.3$ & $-5.48--5.38$ & 1.0 & -5.4 \\
Xexp,min & $-16.4-0.6$ & $-7.38--6.76$ & 3.9 & -7.1 \\
$\Delta \bar{X}$ rs & $-0.8-9.0$ & $2.53-3.12$ & 6.0 & 2.8 \\
Xpeak-to-peak & $0.1-16.7$ & $5.99-6.02$ & 0.2 & 6.0 \\
\hline
\end{tabular}

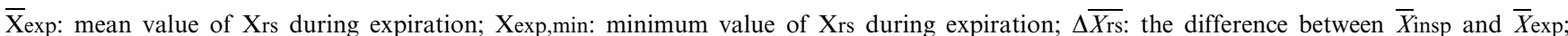
Xpeak-to-peak: the difference between Xinsp,max and Xexp,min. Total range of values assumed by indices, optimal range (range of threshold values in which both specificity and sensitivity were $100 \%$ ), its percentage of the total range (optimal region) and the midpoint of the optimal range (optimal threshold) are reported. Indices are as described in the text. 

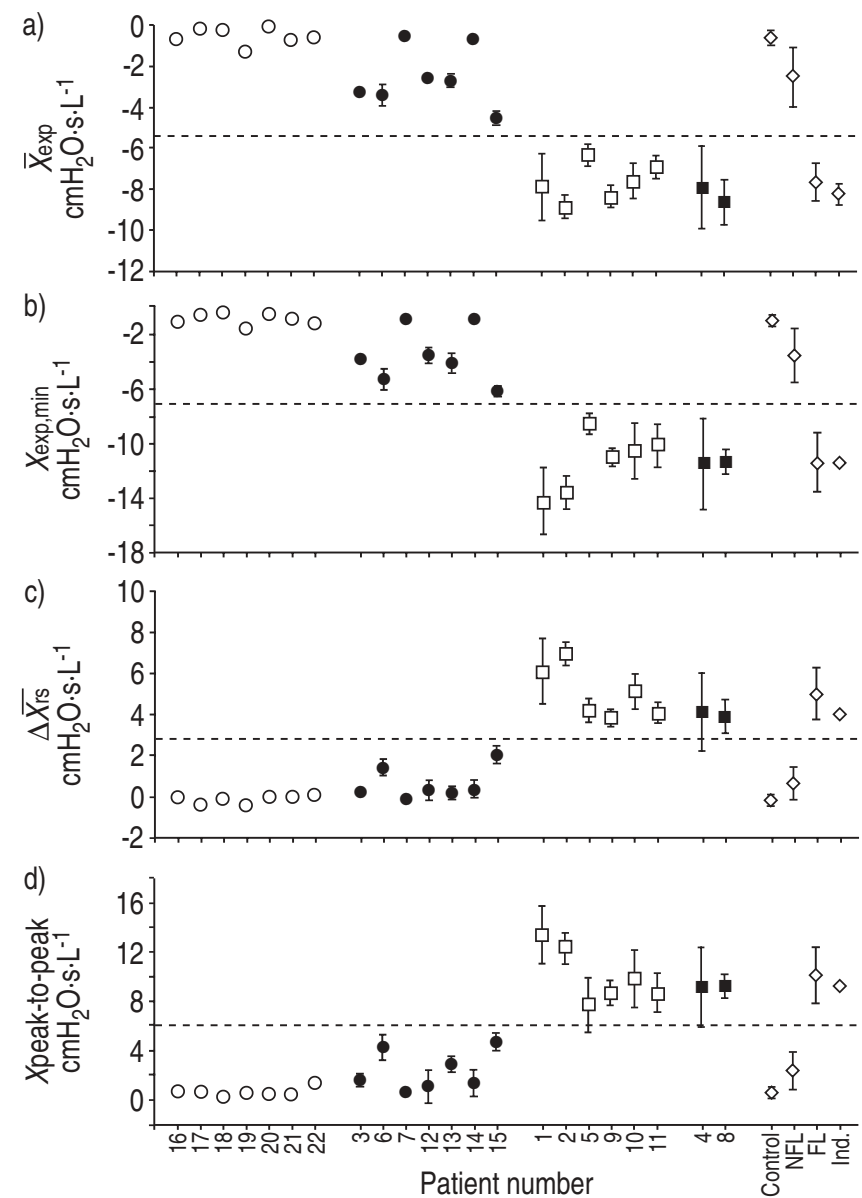

Fig. 5. - Mean \pm SD values of the considered indices computed on all the breaths from a given subject are shown. Control subjects $(\bigcirc)$, nonflow-limited (NFL; - ), flow-limited (FL; $\square$ ) and indeterminate (Ind.) chronic obstructive pulmonary disease patients (ם) and mean \pm SD of each group $(\diamond)$. a) Mean expiratory respiratory system reactance $\left(X_{r s}\right)$ value, b) minimum expiratory $X_{r s}$ value, c) the difference between mean inspiratory and mean expiratory $\mathrm{X}_{\mathrm{rs}}\left(\Delta \overline{X_{\mathrm{rs}}}\right)$ and d) the difference between maximum inspiratory and minimum expiratory $\mathrm{X}_{\mathrm{rs}}$ (Xpeak-to-peak) were considered. In all the graphs, the optimal threshold value (table 2) is plotted as a horizontal dashed line.

opening, thereby increasing $P$ fr, flow limitation is assumed to be present if expiratory flow does not increase. However, NEP was not used as a gold standard, as its assumptions have never been validated by comparison with other physiological measurements of EFL in spontaneously breathing unsedated patients. Instead the $\mathrm{M}-\mathrm{W}$ technique was used; when $\mathrm{Pfr}$ increases and flow decreases, there is a clockwise loop in the expiratory $\mathrm{Pfr} / V^{\prime}$ ao curve that is not seen during inspiration (fig. 6a). This method of detecting EFL in each breath has a number of advantages. It is independent of upper airway compliance that can potentially influence the results of NEP measurements. Like NEP it is not influenced by the previous $V \mathrm{~L}$ history as it does not require specific respiratory maneuvers to be performed. However, it is recognised that it is possible for such a loop to be present when dynamic compression of airways is combined with volume dependence of resistance in the absence of choke points, completely limiting expiratory flow as illustrated in the schematic shown in figure $6 \mathrm{~b}$. In this situation, Xrs would not decrease when, according to the M-W criterion (and NEP), there was EFL. Alternatively, choke points could develop in some parallel
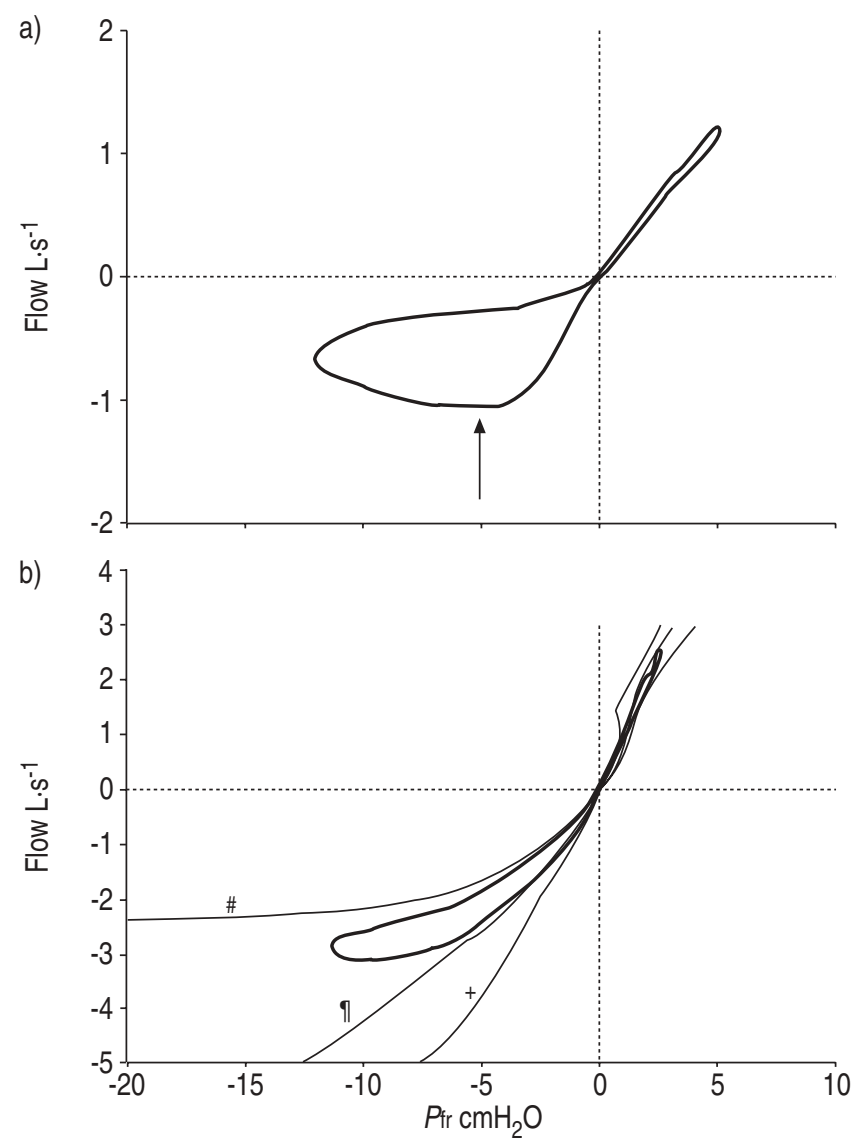

Fig. 6.-Schematic of a) flow-resistive pressure drop ( $P$ fr $)$ versus flow diagram in presence of flow-limitation and b) in case of dynamic compression of airways combined with volume dependence of resistance in the absence of choke points. In a) the arrow indicates the onset of expiratory flow limitation. In b) there are three schematic isovolume pressure/flow curves (thin lines) at $\left.25 \%\left({ }^{\#}\right), 50 \%{ }^{(}\right)$and $75 \%\left({ }^{+}\right)$vital capacity (VC). On the same plot a schematic pressure/ flow loop during a tidal breath is also shown (thick line). This last plot takes into account the increase in resistance as lung volume decreases. Thus, if the expiration starts for instance at $50 \%$ of $\mathrm{VC}$, the quiet-breathing $(\mathrm{QB})$ loop at the beginning will be very close to the isovolume pressure/flow curve at $50 \% \mathrm{VC}$. Then the lung volume will decrease, leading to the separation of the QB loop from the $50 \%$ $\mathrm{VC}$ isovolume curve and then approaches, for instance, the $25 \%$ isovolume curve at the end of expiration. The schematic was drawn by hand and the values of 50 and $25 \%$ of $\mathrm{VC}$ for end inspiration and end expiration are arbitrary and considered only for clarity.

pathways before expiratory flow was completely limited. If this were to occur, the authors predict that the forced oscillations would penetrate to those alveoli where flow was not yet limited, but would not pass through the choke points that were established in parallel. This would cause a fall in Xrs, but not to the extent as would occur when choke points limited all expiratory flow, while NEP should indicate lack of flow limitation.

Ideally, the authors would have preferred a gold standard methodology that only detected complete EFL produced by choke points. In practice, the comparator used encompassed the possibility of no flow limitation with volume dependence of resistance combined with early dynamic compression of airways insufficient to limit flow and also of partial EFL by choke points in some parallel pathways but not in others. Measurement of Xrs is likely to be insensitive to the volumedependent effects, sensitive to complete EFL and intermediate with partial EFL. A false-positive M-W analysis due to early dynamic compression and volume dependence of resistance 

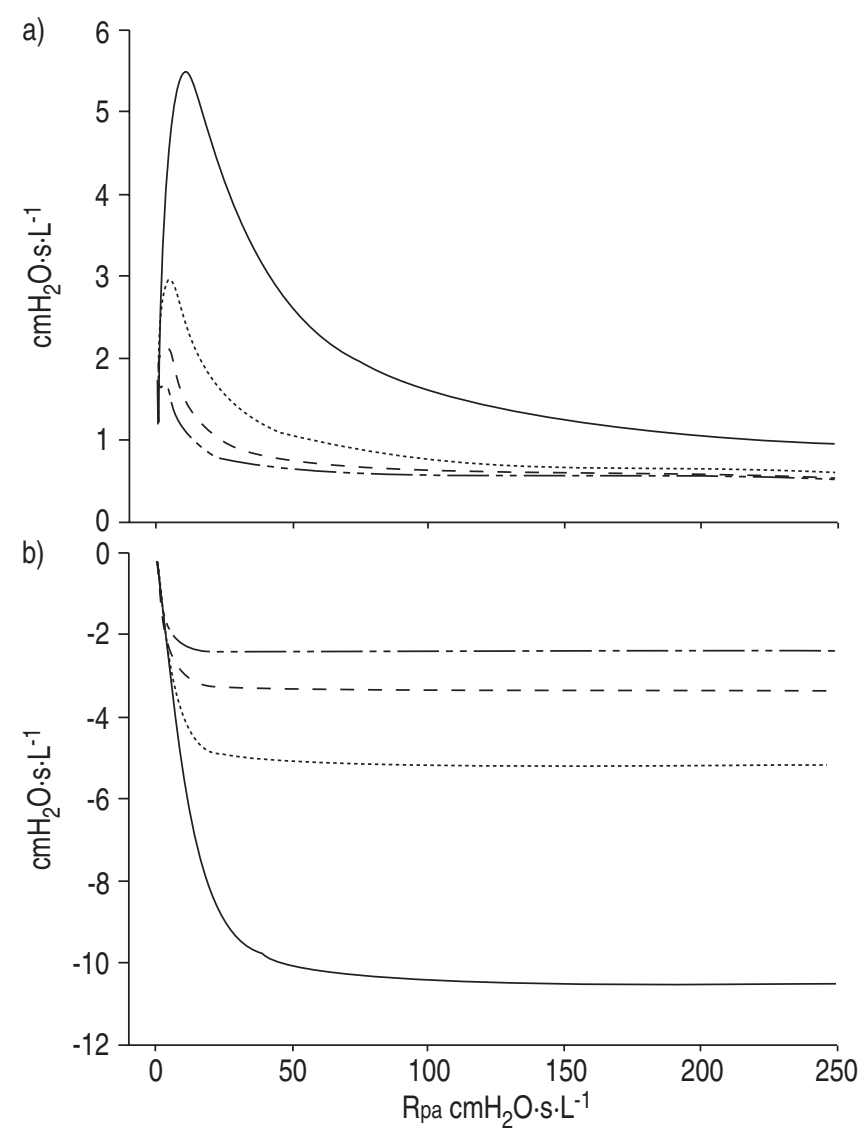

Fig. 7.-Simulated a) real and b) imaginary part of the input impedance at $5(-), 10(\cdots \cdots \cdots), 15\left(-{ }_{-}\right)$and $20\left(-\cdot \cdot{ }^{-} \cdot \cdot\right) \mathrm{Hz}$ of a lumped parameter model of the respiratory system that includes airway wall shunting. Real and imaginary parts are plotted versus peripheral airway resistance $(\mathrm{Rpa})$ that is increased to simulate the occurrence of expiratory flow limitation. See Appendix 1 for details.

would appear as a lack of sensitivity of Xrs indices to detect EFL. As this was never seen over the optimal range of threshold values (table 2 and fig. 4), false-positive results with this method are probably rare.

If partial flow limitation had caused a false-positive M-W analysis that was also detected by Xrs analysis, the sensitivity curves in figure 4 would have reached $100 \%$ earlier, leading to a large optimal range. The fact that these ranges were small for $X$ exp and Xpeak-to-peak argues against this being a significant problem. If Xrs analysis were insensitive to partial EFL it would again reduce the test sensitivity, which was not the case.

The transition from NFL expiration to complete EFL has not been carefully studied. Sophisticated modelling combined with careful physiological measurements during this transition are needed to clarify this issue. The forced oscillation method can help by giving a quantitative estimate of the degree of EFL. Xrs should fall as each new choke point develops by an amount dependent on the elastic properties of that part of the tracheobronchial tree subtended by the airway in which the choke point occurs. However, the degree of Xrs reduction during EFL also depends on the mechanical properties of airway walls (which may be hypercompliant in COPD [22]) and on the location of the choke points. Nevertheless, in this study, the intrasubject variability of airway wall properties or location of choke points did not prevent the definition of a single threshold value (independent from subject characteristics) that reliably indicates the presence or absence of EFL.
In this study, the authors have developed indices from $\mathrm{Xrs}$ measurements that detect EFL robustly. From these indices they were able to identify threshold values, obtained in a limited number of patients, between which sensitivity and specificity were $100 \%$. In a larger patient population it is possible that these thresholds may change somewhat. This clear separation of values also precluded the use of receiver operator characteristic analysis.

Although all the indices detected EFL, each had advantages and disadvantages. $\Delta \overline{X \text { rs }}$ presented the clearest separation between FL and NFL breaths in the sensitivity-specificity plots (fig. 4). Moreover, this index is less dependent on baseline airway mechanics being based on a relative change rather than an absolute value. Indices based on mean values are more robust because they are less affected by signal noise, but must be computed using the whole breath. The index based on the minimum expiratory $\mathrm{Xrs}$ is well suited to study changes in flow limitation during the breath; the point when the actual Xrs value falls below the threshold indicates the onset of flow limitation that persists until Xrs returns to values higher than threshold. This index could be used to detect the $V \mathrm{~L}$ at which EFL occurred (and thus to automatically compute, for instance, the percentage of a tidal breath that is FL) and the relative value of the limiting flow.

In all the analyses the Xrs data was expressed as the absolute values and the relative changes of reactance rather than expressing data as a percentage of baseline (i.e. inspiratory Xrs) [9], since the inspiratory Xrs values can range from slightly positive to negative depending on the mechanical properties of the respiratory system of the subject. However, the use of physical units should not prevent the application of the Xrs thresholds to new patients and data sets. In fact, whatever the condition of the patient, the difference between the impedance of the shunt pathway due to airway wall compliance (measured by expiratory Xrs if EFL is present) and the open lung (measured by Xrs during inspiration) is so high (approximately one order of magnitude) that even differences on airway wall mechanical properties due to intersubject variability or to the disease should only marginally affect the changes in Xrs during expiration. Moreover, as shown in the Appendix and in figure 7, Xrs is very sensitive to the increase of peripheral airways resistance only at the beginning and rapidly reaches a plateau. These observations suggest that the thresholds should only marginally be affected by changes in lung and airways mechanics. This is also supported by the $100 \%$ sensitivity and specificity obtained studying a very heterogeneous patient population (table 1). Finally, even if the oscillatory pressure applied to the subject during FOT was very small $\left(<2 \mathrm{cmH}_{2} \mathrm{O}\right)$ and with a zero mean value (as it was a sinusoidal forcing), it is possible that the dead space and the resistance added by the device may have induced changes in patients' $V \mathrm{~L}$ and breathing pattern. As a result, the condition of the patients during the measurements may have been different from baseline spontaneous breathing. However, the amount of dead space and resistance of the device was similar to any measurement system that uses pneumotachographs (as NEP or spirometers). Therefore, the effect of FOT on breathing pattern is likely comparable with any other EFL monitoring system.

Since changes in Xrs can be due to either EFL or airway closure, it is possible that the swing in Xrs may be due in part to the latter phenomenon, affecting the reliability of the technique to selectively detect EFL. However, as shown in figure 3 , the time course of $\mathrm{Xrs}$ is not in phase with volume. Typically, Xrs should increase from mid- to late expiration, and at end-expiration is close to its value at end-inspiration. This is the expected pattern if $\mathrm{Xrs}$ is detecting flow limitation, which does not persist to the end of expiration but stops before expiratory flow does when pleural pressure falls and 
the airways are no longer dynamically compressed. If airway closure were occurring, it should increase throughout expiration and Xrs would continue to fall until end-expiration and remain low throughout early inspiration before all closed airway reopened. This behaviour was not observed, since $\mathrm{Xrs}$ starts to return to pre-expiratory values before endexpiration. This happens as Poes begins to fall (see fig. 3).

The fall in $P$ oes due to pre-inspiratory inspiratory muscle recruitment necessary to overcome intrinsic positive endexpiratory pressure can decrease dynamic compression so that flow limitation is no longer present. It cannot, however, open airways closed below closing volume as long as $V \mathrm{~L}$ is still decreasing. Reopening of closed airways below closing volume only occurs after inspiratory flow starts and $V \mathrm{~L}$ reaches opening volume. Thus, the increase in Xrs toward the end of expiration must be due to either partial or complete reopening of choke points.

However, a perfect quantification of flow limitation and airway closure contributions to Xrs is very difficult in these circumstances. Nevertheless it is important to underline that it is impossible to differentiate the impact of airway closure and EFL during an expiration using any noninvasive monitoring approaches (including NEP).

These data are in keeping with the theoretical basis for using the negative swing in Xrs to detect EFL. The Xrs value at a given frequency results from two opposite contributions: one negative, related to compliance and one positive related to inertance. Thus, the observed within-breath changes in $\mathrm{Xrs}$ can be due to either a reduction in the apparent compliance and/or a decrease of inertance. Lung and chest wall compliances are functions of volume and not flow, and in the absence of airway closure in expiration and reopening in inspiration or the development of choke points during expiration they should change very little during the respiratory cycle. Inhomogeneities of the time constant can lead to lower values of Xrs in COPD compared with healthy controls (as in fig. 2), but, unless airway closure is occurring, inhomogeneities can only contribute for a small fraction of the changes of Xrs observed in the presence of EFL [23], even if they decrease substantially during inspiration, as a result of the dilatation of the peripheral airways. Respiratory system inertance is mainly due to gas acceleration in the airways [24] and the mass of the abdomen. At $5 \mathrm{~Hz}$ its contribution to total $\mathrm{Xrs}$ is in the order of $0.4 \mathrm{cmH}_{2} \mathrm{O} \cdot \mathrm{s} \cdot \mathrm{L}^{-1}$ [25]. The mean within-breath peak-to-peak difference of $\mathrm{Xrs}$ observed in the FL patients was $10.31 \mathrm{cmH}{ }_{2} \mathrm{O} \cdot \mathrm{s} \cdot \mathrm{L}^{-1}$ (fig. $5 \mathrm{~d}$ ), therefore even the maximum possible decrease of inertance to zero can account for only a negligible part $(\sim 5 \%)$ of the observed reduction. Thus changes of inertance are unlikely to play a significant role in the changes in Xrs that were measured.

In summary, these data indicate that the measurement of expiratory reactance during tidal breathing can reliably detect breaths that are flow-limited and potentially the time at which flow limitation begins. A further useful feature of this method is its ability to identify periods in which total respiratory input resistance no longer reflects the mechanical properties of the respiratory system due to the presence of expiratory flow limitation. This technique is simple to use, sensitive, specific and noninvasive. The ability to analyse multiple breaths in different circumstances makes this a useful method in conditions where flow limitation has been hard to measure, such as during exercise and in the intensive care unit. Moreover, this noninvasive technique is particularly suited to evaluating clinical interventions such as bronchodilator treatment where it will allow the monitoring of more relevant variables than the forced expiratory volume in one second, and potentially identify those patients who benefit most from therapy.

\section{Appendix}

To evaluate the impact of the different forcing frequencies on the Xrs swings observed when passing from FL to NFL conditions the authors modelled the respiratory system as a simple lumped parameter model derived from the twocompartment model proposed by MEAD [26]. The model considers the airways to be compliant structures that may shunt some of the forced oscillatory flow. The present model consisted of an airways compartment in series with parallel alveolar gas compliance and lung-chest wall tissue compartments.

Airways were modelled as an upper airway resistance $\left(0.5 \mathrm{cmH}{ }_{2} \mathrm{O} \cdot \mathrm{s} \cdot \mathrm{L}^{-1}\right)$, an airway inertance $\left(0.002 \mathrm{cmH}_{2} \mathrm{O} \cdot \mathrm{s}^{2} \cdot \mathrm{L}^{-1}\right)$, an airway wall compliance $\left(0.002 \mathrm{~L} \cdot \mathrm{cmH}_{2} \mathrm{O}^{-1}\right)$ shunt pathway and a peripheral airway resistance $(\mathrm{Rpa})$ connected as a $\mathrm{T}$ network. The airways compartment (on the Rpa side) leads to a gas compression compliance (equivalent of $3 \mathrm{~L}$ of air) in parallel with the tissues, modelled as a resistance ( $\mathrm{Rt}$; $0.5 \mathrm{cmH}_{2} \mathrm{O} \cdot \mathrm{s} \cdot \mathrm{L}^{-1}$ ) in series with a compliance ( $\mathrm{CL}$, dyn; $0.05 \mathrm{~L} \cdot \mathrm{cmH}_{2} \mathrm{O}^{-1}$ ) [25]. Using this simple model, the authors simulated the effect of the onset of EFL as the increase of Rpa from a baseline value of 0.5 up to $250 \mathrm{cmH}_{2} \mathrm{O} \cdot \mathrm{s} \cdot \mathrm{L}^{-1}$ [27].

In figure 7 , the real and the imaginary part of the $\mathrm{Zin}$ presented by the model are expressed as a function of Rpa. As soon as Rpa increases the shunt pathway due to the airway walls compliance affects the total input impedance by reducing Xrs. Even when this reduction is present at all the frequencies, the greatest difference is seen at the lowest frequency. Therefore, to obtain larger Xrs swings from inspiration to expiration when expiration is flow-limited (and thus increasing the sensitivity of the indices), the lowest possible frequency was chosen. Since the quiet breathing signal can interfere with the estimation of Zin at frequencies below $5 \mathrm{~Hz}$, $5 \mathrm{~Hz}$ was used as forcing frequency in this study. This forcing frequency allows for a time resolution of $0.2 \mathrm{~s}$ (i.e. one period of the forcing signal).

Figure 7 also shows that the real part of $\mathrm{Zin}$ is not monotonic, with an increase at the beginning followed by a decrease. This suggests that Rrs is not suitable for the detection of EFL as the same value of Rrs can be measured in presence of both a mild or a massive increase in Rpa.

This model is an extremely simplified representation of the respiratory system that considers only one pathway (instead of several heterogeneous airways) with constant values for the several parameters instead of considering possible withinbreath variations. The authors accept that the transition phase between non-EFL to EFL during an expiration is a complex and heterogeneous phenomenon, both in time and in the location and the number of pathways involved; therefore its use is rather speculative. Nevertheless, it was found that the Xrs values measured at different frequencies $(5,11$ and $19 \mathrm{~Hz}$ ) during EFL in a subset of the COPD patients frequency are well represented by this model, when Rpa assumes very high values.

Acknowledgment. The authors are grateful to P. Carlucci for clinical assistance during the experiments and to A. Lo Mauro, A. Iorio and R. Esposti for technical assistance.

\section{References}

1. Olafsson S, Hyatt RE. Ventilatory mechanics and expiratory flow limitation during exercise in normal subjects. J Clin Invest 1969; 48: 564-573.

2. Hyatt RE. The interrelationship of pressure, flow and 
volume during various respiratory maneuvers in normal and emphysematous patients. Am Rev Respir Dis 1961; 83: 676683.

3. O'Donnell DE, Revill SM, Webb KA. Dynamic hyperinflation and exercise intolerance in chronic obstructive pulmonary disease. Am J Respir Crit Care Med 2001; 164: 770-777.

4. Marin JM, Carrizo SJ, Gascon M, Sanchez A, Gallego B, Celli BR. Inspiratory capacity, dynamic hyperinflation, breathlessness, and exercise performance during the 6-minute-walk test in chronic obstructive pulmonary disease. Am J Respir Crit Care Med 2001; 163: 1395-1399.

5. Eltayara L, Becklake MR, Volta CA, Milic-Emili J. Relationship between chronic dyspnea and expiratory flow limitation in patients with chronic obstructive pulmonary disease. Am J Respir Crit Care Med 1996; 154: 1726-1734.

6. Boni E, Corda L, Franchini D, et al. Volume effect and exertional dyspnoea after bronchodilator in patients with COPD with and without expiratory flow limitation at rest. Thorax 2002; 57: 528-532.

7. Peslin R, Felicio da Silva J, Duvivier C, Chabot F. Respiratory mechanics studied by forced oscillations during artificial ventilation. Eur Respir J 1993; 6: 772-784.

8. Peslin R, Farre R, Rotger M, Navajas D. Effect of expiratory flow limitation on respiratory mechanical impedance: a model study. J Appl Physiol 1996; 81: 2399-2406.

9. Vassiliou M, Peslin R, Saunier C, Duvivier C. Expiratory flow limitation during mechanical ventilation detected by the forced oscillation method. Eur Respir J 1996; 9: 779-786.

10. Dawson SV, Elliott EA. Wave-speed limitation on expiratory flow - a unifying concept. J Appl Physiol 1977; 43: 498515.

11. Pauwels RA, Buist AS, Calverley PM, Jenkins CR, Hurd SS. Global strategy for the diagnosis, management, and prevention of chronic obstructive pulmonary disease. National Heart, Lung and Blood Institute/World Health Organization Global Initiative for Chronic Obstructive Lung Disease (GOLD) Workshop summary. Am J Respir Crit Care Med 2001; 163: 1256-1276.

12. Quanjer PH, Tammeling GJ, Cotes JE, Pedersen OF, Peslin, Yernault JC. Lung volumes and forced ventilatory flows. Report Working Party Standardization of Lung Function Tests, European Community for Steel and Coal. Official Statement of the European Respiratory Society. Eur Respir J 1993; 6: Suppl. 16, 5-40.

13. Baydur A, Behrakis PK, Zin WA, Jaeger M, Milic-Emili J. A simple method for assessing the validity of the esophageal balloon technique. Am Rev Respir Dis 1982; 126: 788-791.

14. Brusasco V, Schiavi E, Basano L, Ottonello P. Comparative evaluation of devices used for measurement of respiratory input impedance in different centres. Eur Respir Rev 1994; 4: $118-120$.

15. Grimby G, Takishima T, Graham W, Macklem P, Mead J. Frequency dependence of flow resistance in patients with obstructive lung disease. J Clin Invest 1968; 47: 1455-1465.

16. Mead J, Whittenberger JL. Physical properties of human lungs measured during spontaneous respiration. $J$ Appl Physiol 1953; 5: 779-796.

17. Kaczka DW, Ingenito EP, Lutchen KR. Technique to determine inspiratory impedance during mechanical ventilation: implications for flow limited patients. Ann Biomed Eng 1999; 27: 340-355.

18. Kaczka DW, Barnas GM, Suki B, Lutchen KR. Assessment of time-domain analyses for estimation of low-frequency respiratory mechanical properties and impedance spectra. Ann Biomed Eng 1995; 23: 135-151.

19. Koulouris NG, Valta P, Lavoie A, et al. A simple method to detect expiratory flow limitation during spontaneous breathing. Eur Respir J 1995; 8: 306-313.

20. Ninane V, Leduc D, Kafi SA, Nasser M, Houa M, Sergysels R. Detection of expiratory flow limitation by manual compression of the abdominal wall. Am J Respir Crit Care Med 2001; 163: 1326-1330.

21. Hyatt RE, Schilder DP, Fry DL. Relationship between maximum expiratory flow and degree of lung inflation. $J$ Appl Physiol 1958; 13: 331-336.

22. Macklem PT, Fraser RG, Bates DV. Bronchial pressures and dimensions in health and obstructive airway disease. $J$ Appl Physiol 1963; 18: 699-706.

23. Gillis HL, Lutchen KR. How heterogeneous bronchoconstriction affects ventilation distribution in human lungs: a morphometric model (published erratum appears in Ann Biomed Eng 1999; 27: 411). Ann Biomed Eng 1999; 27: 14 22.

24. Oostveen E, Peslin R, Duvivier C, Rotger M, Mead J. Airways impedance during single breaths of foreign gases J Appl Physiol 1991; 71: 1813-1821.

25. Peslin R, Fredberg JJ. Oscillation mechanics of the respiratory system. In: Macklem PT, ed. Handbook of Physiology. Section 3: The Respiratory System. Bethesda, American Physiological Society, 1986; pp. 145-177.

26. Mead J. Contribution of compliance of airways to frequency-dependent behavior of lungs. $J$ Appl Physiol 1969; 26: 670-673.

27. Vassiliou M, Peslin R, Saunier C, Duvivier C. Expiratory flow limitation during mechanical ventilation detected by the forced oscillation method. Eur Respir J 1996; 9: 779-786. 